\title{
A pulse-tube refrigerator using variable-resistance orifice
}

\author{
B.J. Huang *, B.W. Sun \\ Department of Mechanical Engineering, National Taiwan University, Taipei 106, Taiwan \\ Received 22 July 2002; accepted 16 January 2003
}

\begin{abstract}
In the present study, we propose a new design of orifice pulse-tube refrigerator (VROPT) using a variable-resistance valve to replace the conventional orifice. The variable-resistance orifice (VRO) is basically a high-speed solenoidal valve similar to the fuel jet device widely used in automobile engines. By changing the frequency and periods of ON and OFF of the valve through an electronic device, we can change the flow resistance of the VRO. This thus provides a possibility for an OPT to be controlled on-line during operation. From the results obtained in the present study, we have shown that VROPT is able to achieve on-line control by regulating the duty cycle $d$ or frequency $f_{\mathrm{v}}$ of the VRO. We also show that VROPT will not loss its thermal performance as compared to conventional OPT.
\end{abstract}

(C) 2003 Elsevier Science Ltd. All rights reserved.

Keywords: Cryocooler; Orifice pulse-tube refrigerator; Variable-resistance orifice pulse-tube refrigerator

\section{Introduction}

The performance of orifice pulse-tube refrigerator (OPT) has been greatly improved in recent years through extensive studies of many researchers. Most studies are focused on the thermal performance improvement using innovative design, for example, double-inlet design [1-4], inertance design [5], use of advanced regenerator material [6], use of symmetrynozzle [7], etc. However, little attention has been paid to the controllability of OPT.

An accurate temperature control at the cold-end of OPT operated under variable environmental conditions is sometimes very important in many applications. The conventional design of OPT provides very little flexibility to this need.

As is well understood, the performance of an OPT is sensitively affected by the flow resistance of the orifice. Researchers tried to make an optimum design of OPT by searching the best orifice (or needle valve) resistance according to the application requirement (cooling load and cold-end temperature) and the compatibility with the other components (reservoir, pulse-tube, regenera-

\footnotetext{
${ }^{*}$ Corresponding author. Tel.: +886-2-2363-4790; fax: +886-2-23640549 .

E-mail address: bjhuang@seed.net.tw (B.J. Huang).
}

tor, etc.). Once the OPT has been designed and fabricated, an on-line adjustment for optimum performance is very difficult.

In the present study, we propose a new design of OPT (or called "variable-resistance orifice pulse-tube refrigerator", VROPT) using a variable-resistance valve to replace the conventional orifice. The variable-resistance valve or orifice (VRO) is basically a high-speed solenoidal valve that is similar to the fuel jet device widely used in automobile engines. By changing the frequency and the periods of $\mathrm{ON}$ and OFF of the valve through an electronic device, we can change the flow resistance of the VRO. This thus provides a possibility for an OPT to be controlled on-line during operation (Figs 1 and 2).

The present study intends to build the new refrigerator to test and demonstrate the feasibility of this new design concept.

\section{Design and test of variable-resistance orifice}

Shown in Fig. 3 is the variable-resistance (VR) valve ("jet nozzle" used in automobile engine, Mitsubishi MD319792) and its actuating circuit. A PC is used to generate a square wave signal to drive the power amplifier through the digital I/O interface device (DI/DO). The valve frequency and the periods of $\mathrm{ON}$ and $\mathrm{OFF}$ thus can be controlled. 


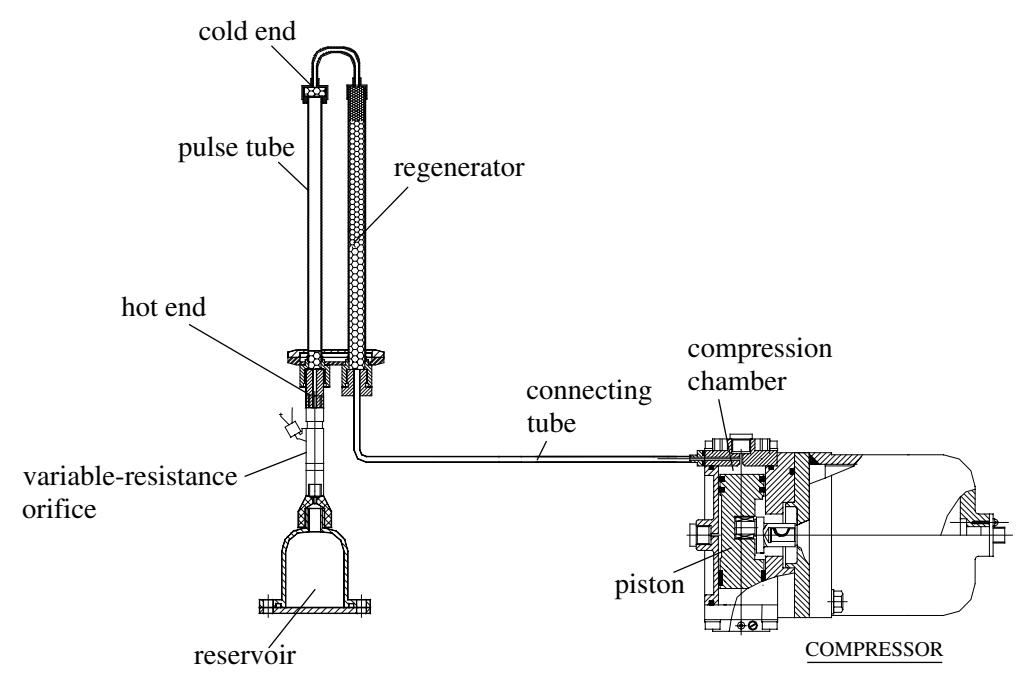

Fig. 1. Schematic diagram of pulse-tube refrigerator with variable-resistance orifice (VROPT).

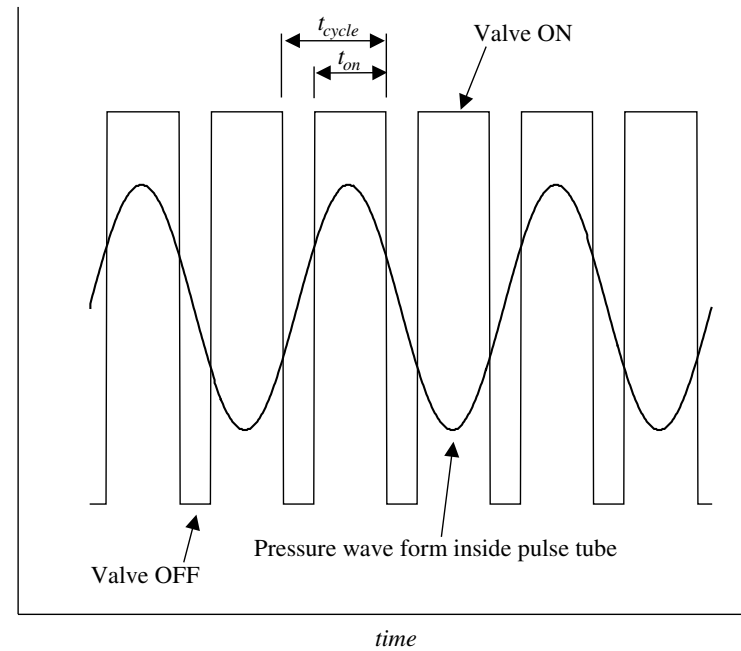

Fig. 2. Valve motion and pressure wave form inside the pulse-tube of a VROPT.

In order to test the flow resistance of VR valve, we built a test rig as shown in Fig. 4.

A steady-state helium flow passes through the VR valve installed in the test section. The pressure drop across the VR valve is measured using two pressure
$Q_{\mathrm{L}} \quad$ cooling load at cold-end, W

$t_{\text {cycle }} \quad$ period of valve motion, $\mathrm{s}$

$t_{\text {on }} \quad$ period of valve opening, $\mathrm{s}$

$T_{\mathrm{L}} \quad$ cold-end temperature, $\mathrm{K}$

$T_{\mathrm{L} 0} \quad$ cold-end temperature at zero cooling load, $\mathrm{K}$

$\begin{array}{ll}P_{\text {co }} & \text { compressor exit pressure, } \mathrm{kg} \mathrm{cm}^{-2} \\ P_{\text {pto }} & \text { gas pressure at hot-end (near pulse-tube), }\end{array}$ $\mathrm{kg} \mathrm{cm}^{-2}$ abs

transducers. The helium mass flow rate is measured using the gas displacement method. The VR valve is actuated by the electronic circuit shown in Fig. 3. In order to compare the performance difference, a needle valve (SS22RS2) which will be used in OPT is also tested in the present study.

Figs. 5 and 6 are the pressure drop curves of the VR valve at different ON/OFF frequency and different duty cycle of valve $\mathrm{ON}, d$, which is defined as

$d=\frac{t_{\text {on }}}{t_{\text {cycle }}}$

where $t_{\mathrm{on}}$ is the period of valve $\mathrm{ON}$, and $t_{\text {cycle }}$ is the period of a complete cycle $=1 / f_{\mathrm{v}}$ and $f_{\mathrm{v}}$ is the ON/OFF frequency of the VR valve. The VR valve reveals an unsymmetrical flow resistance depending on flow direction, especially at higher $d$. At $f_{\mathrm{v}}>15 \mathrm{~Hz}$, the pressure drop at reverse flow condition (from reservoir toward pulse-tube) is larger than that at forward flow (from pulse-tube toward reservoir) by $60-90 \%$. This is due to the fact that the VR valve used in the present study is mainly for automobiles as a fuel jet nozzle. The nozzle shape design makes the VR valve behaves unsymmetrical characteristics on flow resistance with flow direction. 


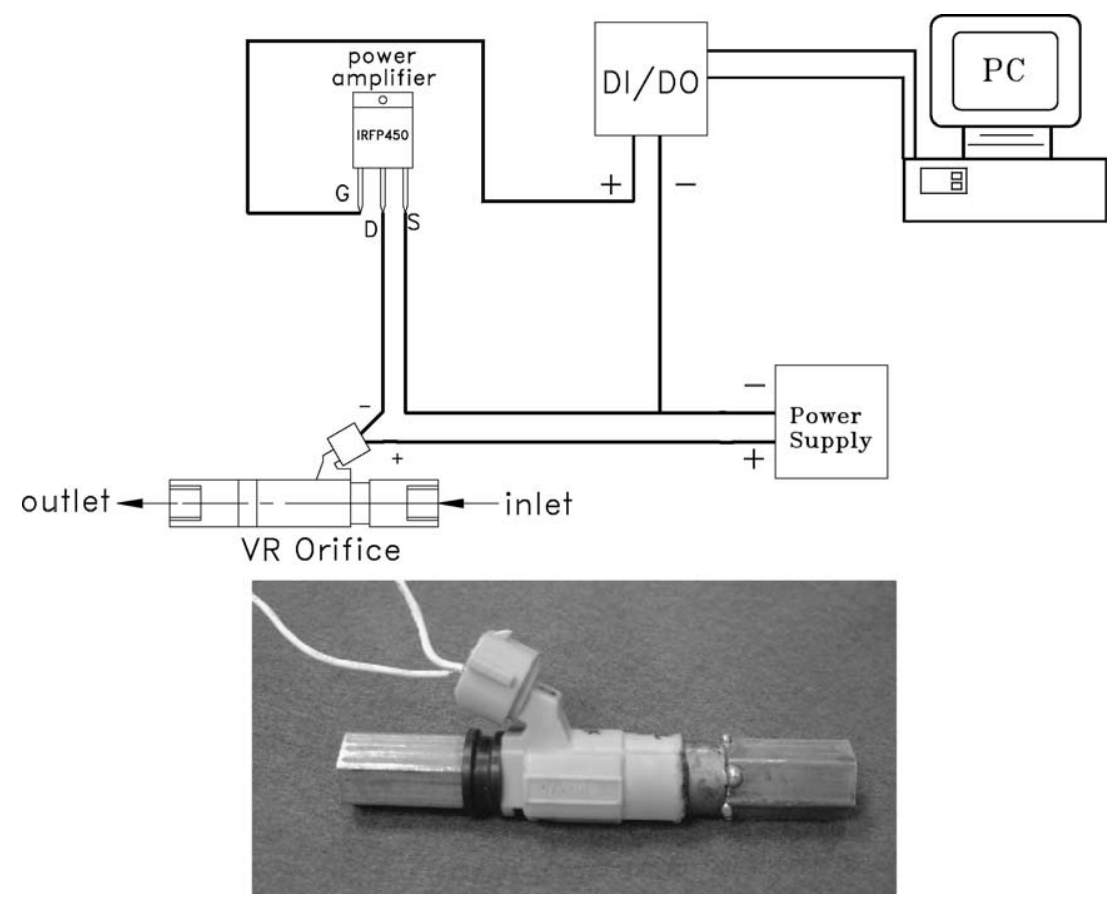

Fig. 3. VR valve (nozzle used in automobile jet engine, Mitsubishi MD319792) and its actuating circuit.

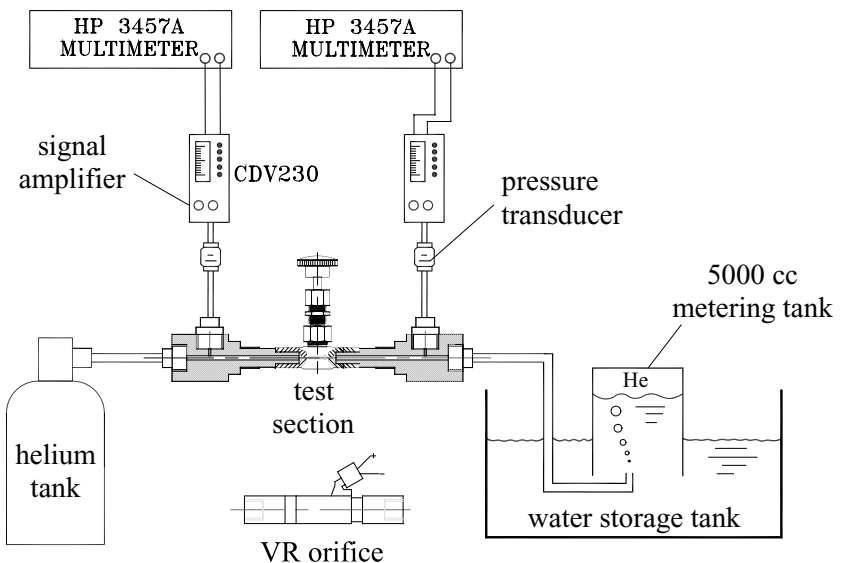

Fig. 4. Schematic diagram of test rig for flow resistance measurement.

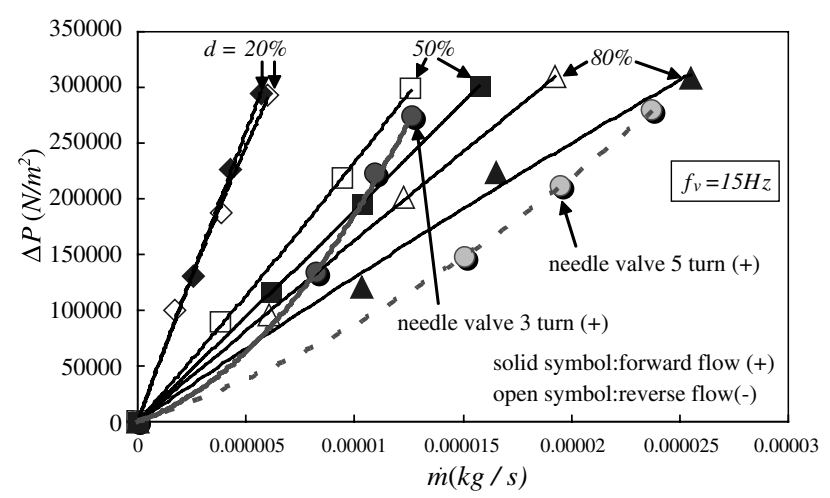

Fig. 5. Pressure drop of VR valve at $15 \mathrm{~Hz}$.

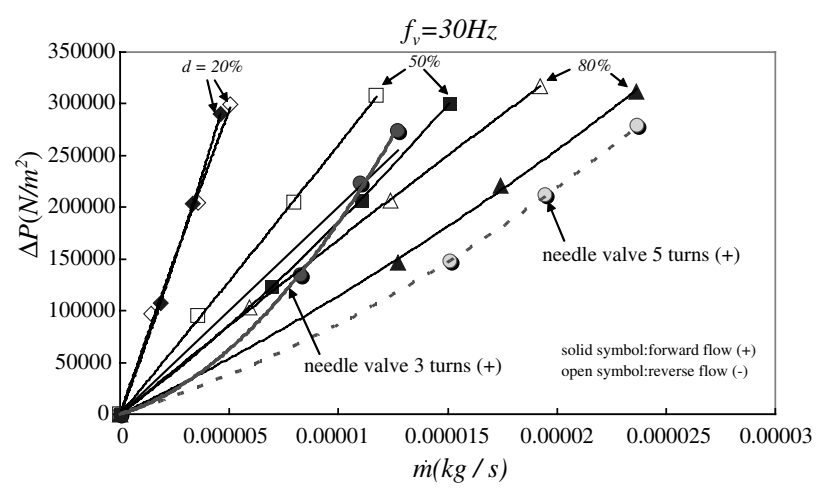

Fig. 6. Pressure drop of VR valve at $30 \mathrm{~Hz}$.

It is noticeable that the pressure drop of VR valve increases linearly with increasing mass flow rate, as compared to the needle valve which has a parabolic relation. For needle valve, the flow resistance is approximately the same in either flow direction, as shown in Fig. 7.

It can be seen that the flow resistance of the VR valve used in the present study increases with valve frequency $f_{\mathrm{v}}$. And, the higher the duty cycle $d$, the lower the flow resistance. Hence, the VR valve provides a means for on-line flow resistance adjustment of OPT.

\section{VROPT refrigerator design and test facility}

Two refrigerators (VROPT3 and VROPT4) were designed and fabricated in the present study. Their 


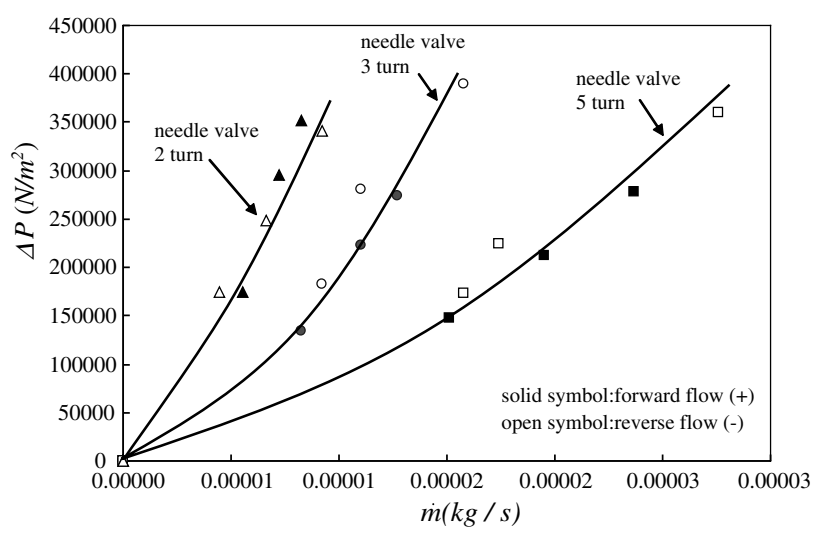

Fig. 7. Pressure drop of needle valve. dimensions are shown in Table 1. For comparison in performance, the VRO of the two refrigerators were changed into the needle valve (SS22RS2) during the experiment. The two refrigerators then become OPT3 and OPT4. All the refrigerators are charged with pure helium gas $(99.99 \%)$ at $15 \mathrm{~kg} / \mathrm{cm}^{2}$ abs and the compressor frequency $f_{\text {opt }}$ is fixed at $10 \mathrm{~Hz}$. A helium compressor with dry-lubrication piston and ceramic cylinder, which was developed in the laboratory, was used [8]. The swept volume of the piston is $16 \mathrm{~cm}^{3}$. We adjusted the DC voltage supplied to the compressor to control the frequency of the piston motion.

The instrumentation of the test rig for measuring the performance of VROPT is shown in Fig. 8. For measuring temperature, T-type thermocouples are used with

Table 1

Design specification of VROPT

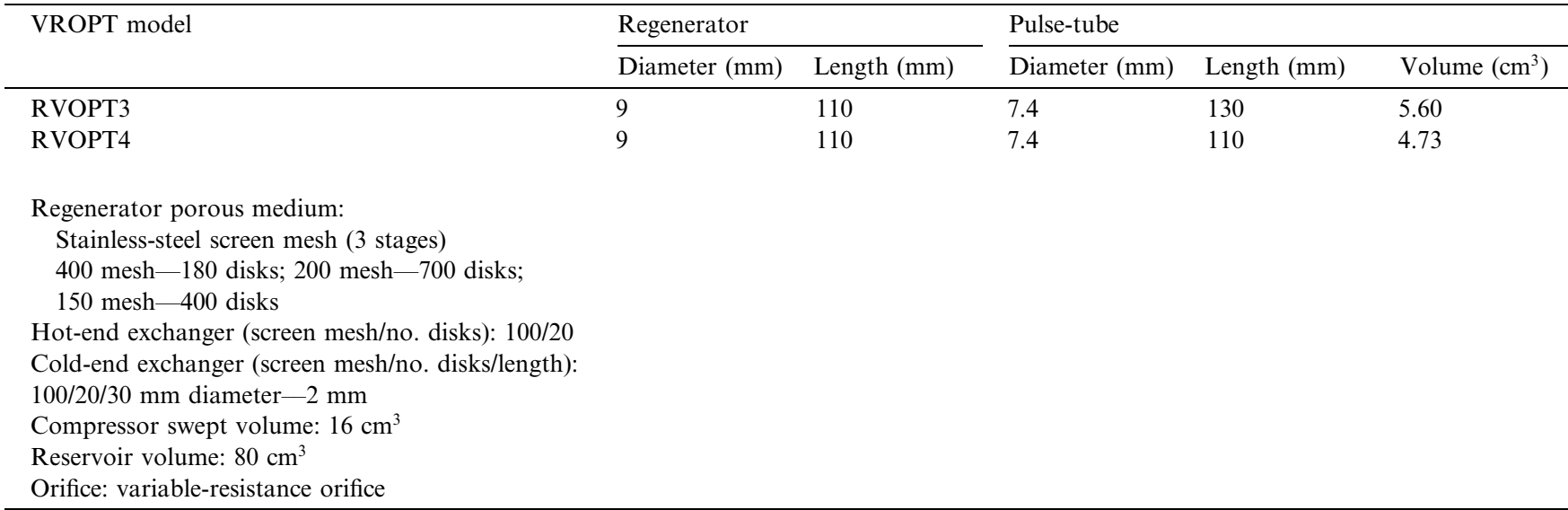

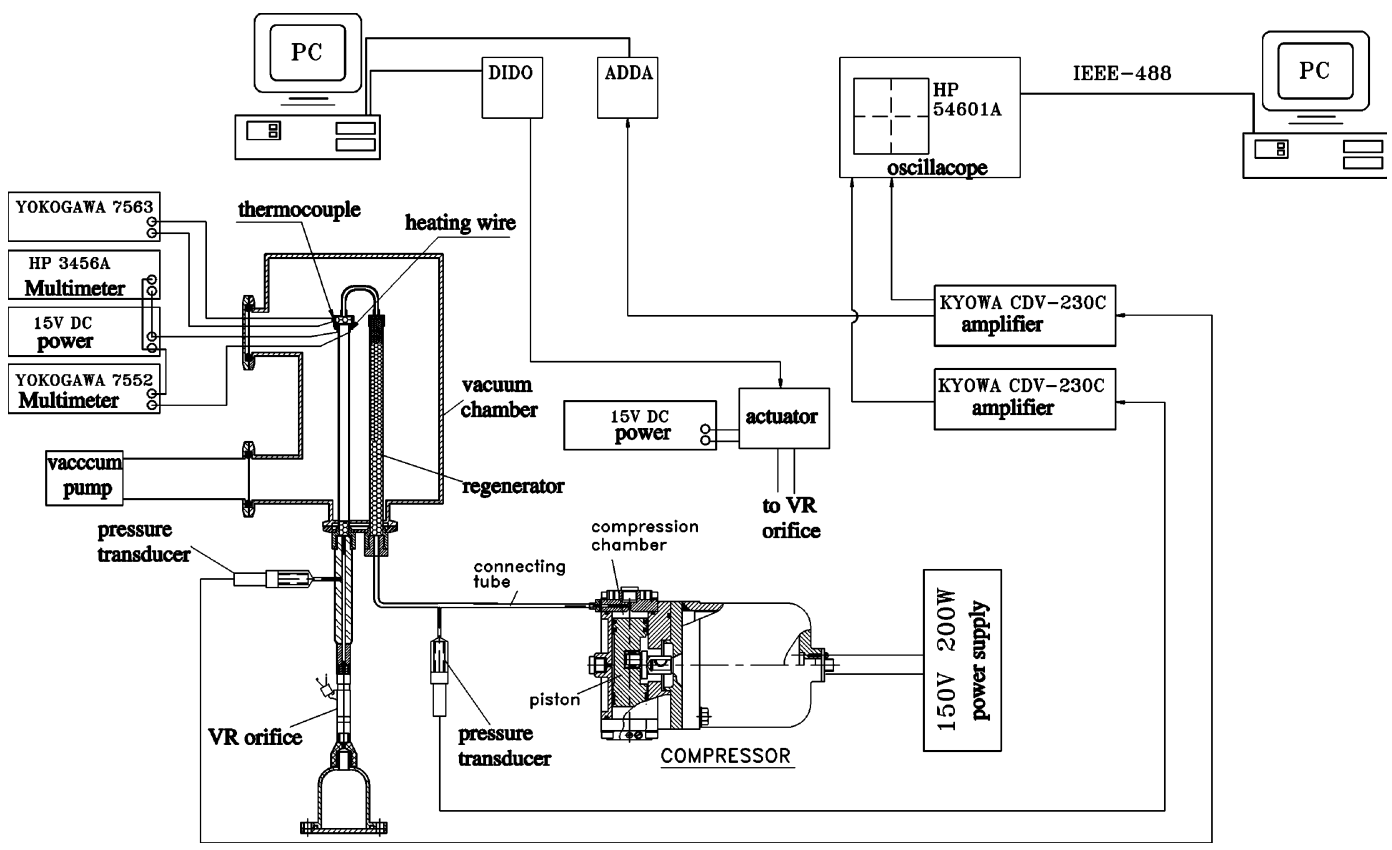

Fig. 8. Schematic diagram of test facility for VROPT. 
digital recorder YOKOGAWA 7563. Two pressure transducers (PGM-50KD, Kyowa Co.) were installed to measure the time-variation pressure at the hot-end exchanger $\left(P_{\mathrm{pto}}\right)$ and the exit of the compressor $\left(P_{\mathrm{co}}\right)$. The cooling load is emulated using a $100 \Omega(15 \mathrm{~cm}$ long $)$ electrical heater wire wounded on the cold-end surface. The power input is supplied by a DC power supply.

In order to reduce the heat leakage to the cold-end and affect the experimental accuracy, the whole VROPT is put into a vacuum chamber that is kept at $10^{-5}$ Torr using a turbo-molecular vacuum pump.

\section{VROPT test results}

The first test was run at zero cooling condition $\left(Q_{\mathrm{L}}=0\right)$ and at pulse-tube operating frequency $f_{\text {opt }}=10$ Hz. Fig. 9 shows that the cold-end temperature at zero cooling load, $T_{\mathrm{L} 0}$, decreases with increasing duty cycle of VR valve, $d$, monotonically, for both VROPT3 and VROPT4. This indicates that the performance of VROPT can be sensitively changed by changing the duty cycle of the VR valve. As to the effect of ON/OFF frequency of VR valve, there seems to be an optimum $f_{\mathrm{v}}$ at which $T_{\mathrm{L} 0}$ reaches the lowest. This is similar to conventional OPT. the optimum $f_{\mathrm{v}}$ is related to the design of VROPT components. For VROPT3, this phenomenon is very clear. But for VROPT4, $T_{\mathrm{L} 0}$ is almost identical for all $f_{\mathrm{v}}$ at $f_{\mathrm{v}}>30 \mathrm{~Hz}$ though $T_{\mathrm{L} 0}$ has a slightly lower value at $f_{\mathrm{v}}=30 \mathrm{~Hz}$ and $d=80 \%$.
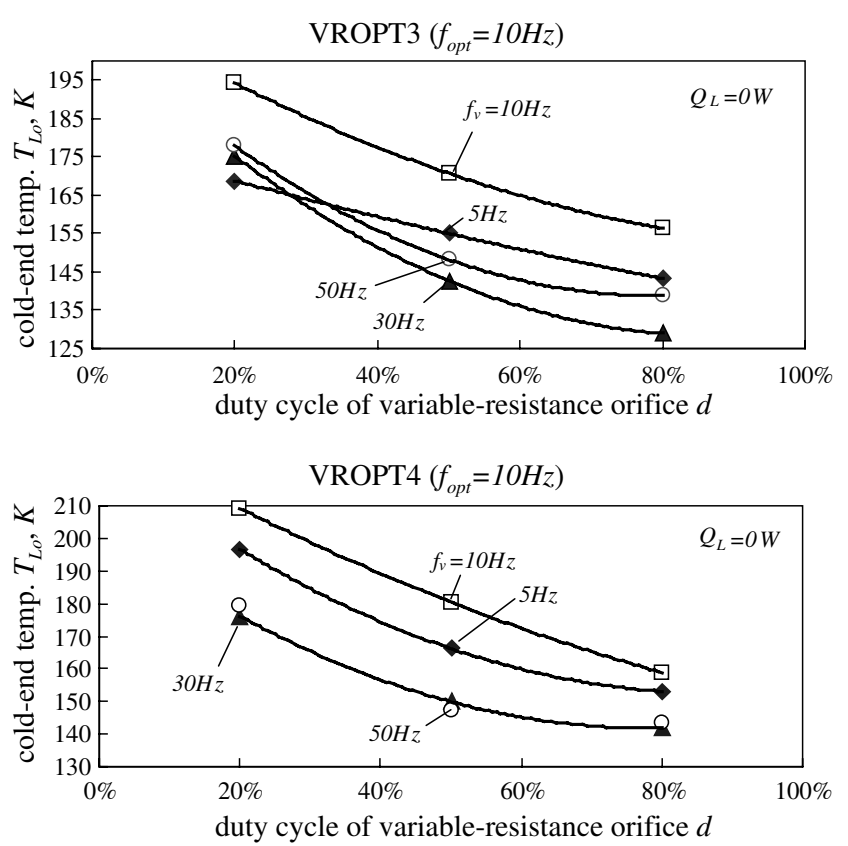

Fig. 9. Variation of zero-load cold-end temperature with duty cycle and valve frequency.
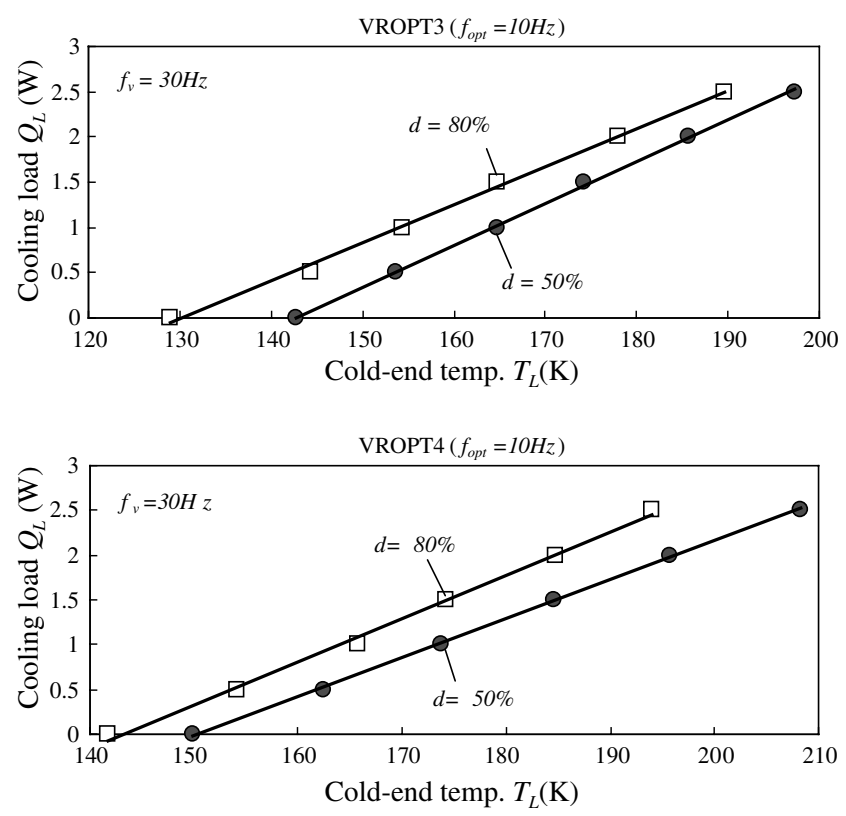

Fig. 10. Variations of cooling load with cold-end temperature at different duty cycle of VRO.

Fig. 10 shows the test results of cooling load variation with cold-end temperature at different duty cycle of VRO $(50 \%$ and $80 \%)$. VROPT3 performs better than VROPT4. It seems that larger duty cycle $d$ has a better performance.

The test results of Figs. 9 and 10 has shown that the online performance control of VROPT is feasible by changing the valve frequency $f_{\mathrm{v}}$ or duty cycle $d$. The other thing needing to be clarified is the possible performance decrease of VROPT as compared to OPT. For this purpose, we tested another two OPT (OPT3 and OPT4). Both refrigerator designs are all the same as VROPT3 and VROPT4, except OPT3 and OPT4 used the needle valve. The test results shown in Fig. 11 indicate that OPT performs better than VROPT at lower duty cycle $(50 \%)$. However, the performance of VROPT is getting closer to OPT as the duty cycle of VR valve increases. The performance of VROPT3 at $d=80 \%$ is very close to that of OPT3.

\section{Discussions and conclusion}

The performance of OPT 3 and OPT4 for three turns and four turns in needle valve adjustment are almost identical as can be seen from Fig. 11. The only design difference of OPT3 and OPT4 is the length of the pulsetube. This means that the performance of OPT is insensitive to the length of pulse tube for the assembly with other components used in the present study. For VROPT, VRO causes some performance decrease and also causes the performance sensitivity with pulse-tube 

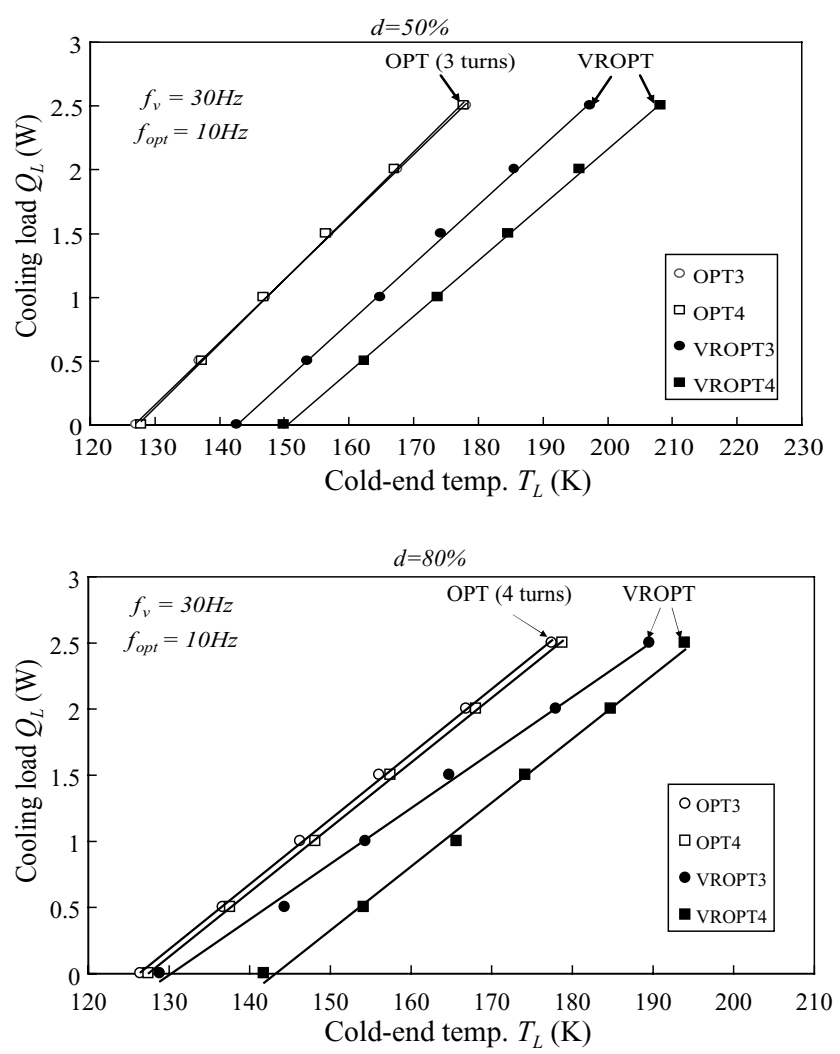

Fig. 11. Performance comparison of OPT and VROPT.

length as compared to OPT. But, at higher duty cycle $d$, the performance of VROPT raises and approaches OPT as shown in Fig. 11.

A possible reason for performance difference between OPT and VROPT is the inconsistency of the orifice flow resistance curves. That is, the flow resistance curves of the valves used in both OPT and VROPT are not exactly identical. This may lead to an incorrect performance comparison of OPT and VROPT. The needle valve (SS22RS2) used in the present OPT has a parabolic relation in pressure drop vs. mass flow rate (Figs. 5 and 6). For forward flow direction (from pulse-tube toward reservoir), the flow resistance of needle valve with three turns is close to that of the VR valve at duty cycle $50 \%$ and valve frequency $30 \mathrm{~Hz}$, at mass flow rate near $0.00001 \mathrm{~kg} / \mathrm{s}$. For four turns in needle valve, the forward flow resistance is close to that of the VR valve at duty cycle $80 \%$ and valve frequency $30 \mathrm{~Hz}$, at low flow rate $(<0.000012 \mathrm{~kg} / \mathrm{s}$, by interpolation from Fig. 6).

The other reason that could cause a lower performance of VROPT is the unsymmetrical flow resistance of the VR valve used in the present experiment. The higher flow resistance in the reverse flow direction (from the reservoir to the pulse-tube) may retard the gas expansion process. This may also explain why the better performance always takes place at higher duty cycle $d$, i.e. longer compression process. Further investigation is necessary in designing a new VR valve with symmetrical flow resistance in either flow direction order to improve the performance. From the present study, it is believed that the thermal performance of VROPT can be very close to or even better than OPT if the VR valve is carefully re-designed.

The ON/OFF control of the VR valve may cause the gas pressure and flow to change inside the pulse-tube. The gas pressure measurements made at the compressor exit and at the hot-end that is near the pulse-tube have shown that the pressure wave forms still approximate sinusoidal shape and are not influenced by the ON/OFF of the VR valve, see Fig. 12.

The VR valve can be designed according to the fuel jet nozzle of automobile engines. The reliability thus can be very high. The size of VR valve can be very small and can be noiseless by using conventional noise insulation technique.

From the results obtained in the present study, we have shown that VROPT is able to achieve on-line control by regulating the duty cycle $d$ or frequency $f_{\mathrm{v}}$ of the VR valve. The configuration of feedback control system for $T_{\mathrm{L}}$ is as shown in Fig. 13. Microprocessor can be implemented in the control system design. A further study is necessary for the optimum design of VROPT at

$f_{v}=30 \mathrm{~Hz} \quad f_{\text {opt }}=10 \mathrm{~Hz} \quad P_{c h}=15 \mathrm{~kg} / \mathrm{cm}^{2}$ abs

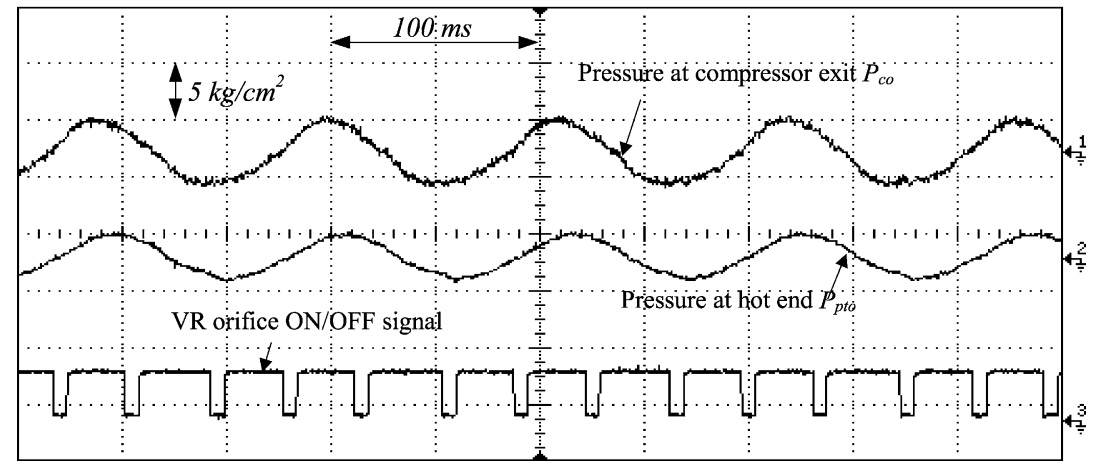

Fig. 12. Pressure waves and ON/OFF signal of VR valve in VROPT3. 


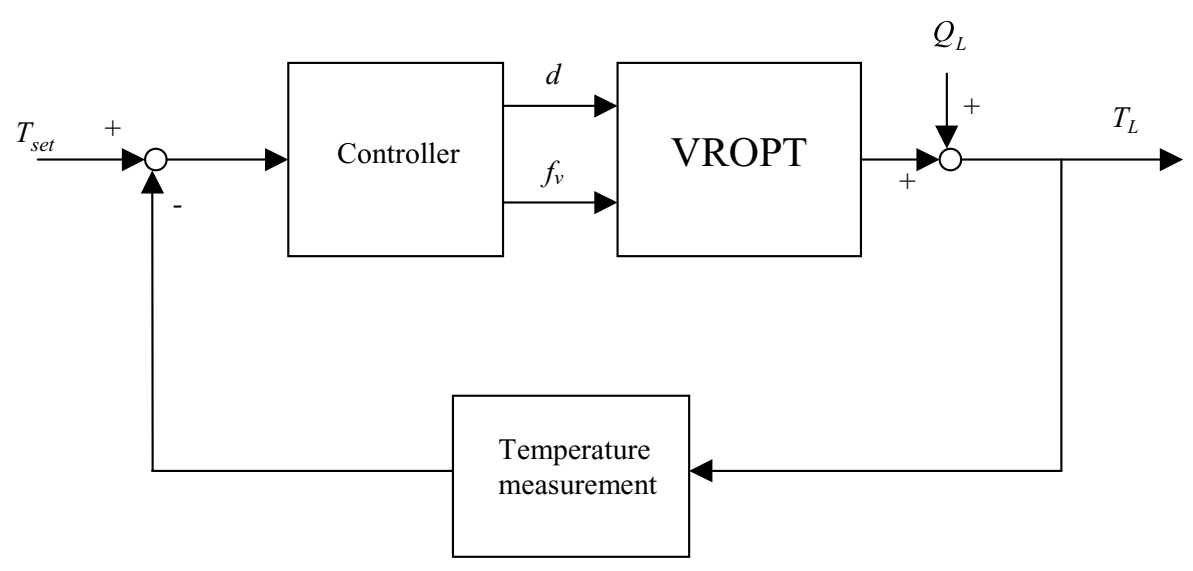

Fig. 13. Control system configuration of VROPT.

various operating conditions and for the microprocessor control system development.

\section{Acknowledgement}

The present study was supported by National Science Council, Taiwan, through Grant no. NSC89-2212-E002-148.

\section{References}

[1] Cai JH, Zhou Y, Wang JJ, Zhu WX. Experimental analysis of double-inlet principle in pulse tube refrigerators. Cryogenics 1993; 33(5):522-5.
[2] Wang C, Wu PY, Chen ZQ. Theoretical and experimental studies of a double-inlet reversible pulse tube refrigerator. Cryogenics 1993;33(6):648-52.

[3] Wang C, Thummes G, Heiden C. Control of DC gas flow in a single-stage double-inlet pulse tube cooler. Cryogenics 1998;38(8): 843-7.

[4] Yang L, Zhou Y, Liang J. Research of pulse tube refrigerator with high and low temperature double-inlet. Cryogenics 1999;39:417-23.

[5] de Boer PCT. Performance of the inertance pulse tube. Cryogenics 2002;42:209-21.

[6] Qiu LM, Numazawa T, Thummes G. Performance improvement of a pulse tube cooler below $4 \mathrm{~K}$ by use of $\mathrm{GdAlO}_{3}$ regenerator material. Cryogenics 2001;41:693-6.

[7] Yang L, Zhou Y, Liang J, Zhu W. Analytical study of the performance of pulse tube refrigerator with symmetry-nozzle. Cryogenics 1999;39:723-7.

[8] Huang BJ, Yu GJ. Experimental study on the design of orifice pulse tube refrigerator. Int J Refrigeration 2001;24:400-8. 